4

\title{
Comparative Infectivity and Transmissibility Studies of Wild- bird and Chicken-Origin Highly Pathogenic Avian Influenza Viruses H5N8 in Chickens
}

\author{
Mohammed A. Rohaim ${ }^{1}$, Rania F. El Naggar², Yehia Madbouly ${ }^{3}$, Mohammed A. \\ AbdelSabour $^{3}$, Kawkab A. Ahmed ${ }^{4}$, Muhammad Munir ${ }^{5 *}$
}

${ }^{1}$ Department of Virology, Faculty of Veterinary Medicine, Cairo University, 12211 Giza, Egypt

${ }^{2}$ Department of Virology, Faculty of Veterinary Medicine, University of Sadat City, 32897 Sadat, Egypt;

${ }^{3}$ Veterinary Serum and Vaccine Research Institute, Abbassia, Cairo 11381, Agricultural Research Center (ARC), Egypt

${ }^{4}$ Department of Pathology, Faculty of Veterinary Medicine, Cairo University, 12211 Giza, Egypt

${ }^{5}$ Division of Biomedical and Life Science, Lancaster University, LA1 4YG Lancaster, Lancashire, UK

"Corresponding Author: Muhammad Munir, muhammad.munir@lancaster.ac.uk; $+441524595083$ 


\section{Abstract}

Despite the recent advances in avian influenza virus surveillance and genomic data, fundamental questions concerning the ecology and evolution of these viruses remain elusive. In Egypt, the H5N8 highly pathogenic avian influenza viruses (HPAIVs) are co-circulating simultaneously with HPAIVs of subtypes H5N1 and low-pathogenic avian influenza viruses (LPAIVs) of subtype H9N2 in both commercial and backyard poultry. In order to isolate AIV from wild birds and to assess their potential in causing infection in commercial poultry, a total of thirty-four cloacal swab samples were collected from apparently healthy migratory wild birds (Anas acuta, Anas crecca, Rallus aquaticus, and Bubulcus ibis) from four Egyptian Governorates (Giza, Menoufia, Gharbia, and Dakahlia). Based on matrix (M) gene-targeting real-time reverse transcriptase PCR and subsequent genetic characterization, our results revealed two positive isolates $(2 / 34)$ for $\mathrm{H} 5 \mathrm{~N} 8$ whereas no $\mathrm{H} 5 \mathrm{~N} 1$ and $\mathrm{H} 9 \mathrm{~N} 2$ subtypes were detected. Genetic characterization of the full-length haemagglutinin (HA) genes revealed the clustering of two reported isolates within group B of clade 2.3.4.4. The potential of a wild birdorigin $\mathrm{H} 5 \mathrm{~N} 8$ virus isolated from a cattle egret for its transmission capability within and between chickens was investigated in compare to chicken origin H5N8 AIV. Chickens inoculated with cattle egret isolate showed varying clinical signs and detection of virus shedding. In contrast, the contact chickens showed less levels of virus secretion indicating efficient virus inter/intraspecies transmission. These results demonstrated the possibility of spread of wild bird origin H5N8 viruses between chicken. In conclusion, our study highlights the need for continuous and frequent monitoring of the genetic diversity of H5N8 AIVs in wild birds as well as commercial poultry sectors for better understanding and determining the genetic nature of these viruses, which is fundamental to predict any future threat through virus reassortment with the potential to threaten human and animal health. Likewise, an assessment of coverage and efficacy of different vaccines and or vaccination regimes in the field conditions should be reconsidered along with strict biosecurity measures.

Keywords: Influenza virus; H5N8; Transmission; Wild birds; Chicken 


\section{Introduction}

Avian influenza is a highly contagious poultry disease, which continues to spread in bird populations throughout the world. Occasionally, a serious public health problem is caused by the transmission of highly pathogenic avian influenza virus (HPAIV) from infected poultry to humans [1]. Highly pathogenic avian influenza virus (HPAIV) subtype H5N8 was first detected in live bird markets in China in 2010 [2, 3]. By the end of 2014, it appeared in Central Asia [4] and reached South Africa arguably by wild migratory birds by July 2017 [5]. Since then multiple spillover events have been attributed to localized outbreaks in commercial and backyard poultry, hobby birds and zoological collections resulting in sever economic losses $[6]$.

The H5N8 viruses in clade 2.3.4.4 were previously diversified into two main groups; Group A and Group B [7]. During the evolution of H5Nx viruses of clade 2.3.4.4, frequent reassortment events have been occurring with other co-circulating HPAIVs and low pathogenicity AIVs in Russia [8], Germany [9], India [10], Italy [11] and Egypt [12]. In Egypt, H5N8 subtype of clade 2.3.4.4 (Group B) was first detected in migratory wild birds during winter season of 2016-2017 [12, 13]. Thereafter, several H5N8 outbreaks have been documented in domestic poultry and ducks in several Egyptian Governorates [14- 21]. A recent study has shown that clade 2.2 viruses are being steadily replaced by clade 2.3.4.4 viruses (H5N8) and reassorting with H9N2 viruses while zoonotic transmission to human has not been reported [22]. Reassortment is a common signature within clade 2.3.4.4 viruses has been reported in different continents including Asia [23], North America [24, 25] and Europe [7] while wild birds were blamed to be responsible for this reassortment [7]. The key factor for clade 2.3.4.4 viruses dissemination is mainly dependent on the flyways of wild birds [6, 7].

Avian influenza viruses (AIVs) are major respiratory pathogens in Egypt's poultry industry and their continuous circulation is related to tremendous socio-economic losses [26]. Continued adaptation and enhanced replication through point mutations for avian influenza viruses are reported. However, no re-assortment in mammalian hosts have been recorded in Egypt [18]. Clades 2.3.4.4b H5N8 viruses have been diversified into at least 5 genotype (Gt1Gt5) as a result of continuous evolution and reassortment; Gt-1 and -2 were detected in 2016 (all in wild birds), Gt-3 and -4 in 2017 (poultry only), and Gt-5 since 2017 (poultry only) [20]. Likewise, the possibility of emergence of novel reassortant H5N8 HPAIVs with unstable gene constellations and zoonotic significance is a public concern [21]. Continuous evolution of avian 
influenza viruses has notoriously been linked to Egypt mainly due to two main hot spots; North Mediterranean Coast that is considered a vital stopover for migratory birds during the annual migration and the Nile Delta region, which is a habitat of an impressive number of bird species [27]. Moreover, introduction of HPAIV subtype H5N8 to the Egyptian poultry populations complicated the disease prevention and control, especially due to endemicity of HPAIV (H5N1) of clade 2.2.1.2 and low pathogenicity AIV (H9N2) strains of G1 lineage [28].

While multiple attempts have been made to report the prevalence of H5N8 AIVs in Egyptian wild birds, information related to clinico-pathological assessment and pathogenicity remained elusive in chickens. This study was carried out to characterize two H5N8 viruses isolated from wild birds during 2019 and their relationships with other contemporary H5N8 viruses to better understand the role of wild birds in avian influenza epidemiology. Studying the clinical pathogenesis of wild bird-origin H5N8 AIVs could also improve the diagnostic aspects of disease and implementation of the control measures. Therefore, the aim of the current study is to investigate the infectious potential and transmission patterns of wild birdorigin $\mathrm{H} 5 \mathrm{~N} 8 \mathrm{AIV}$ in commercial poultry to underpin the potential virus spillover from wild birds to poultry.

\section{Materials and Methods}

\subsection{Ethics statement, Samples collection, Virus isolation and Genetic Characterization}

All animal studies and procedures were carried out under strict accordance with the guidance and regulations of animal welfare and health. As part of this process, the work approved by the Ethics Committee at Veterinary Serum and Vaccine Research Institute (VSVRI), Agricultural Research Center (ARC), Abbassia, Cairo, Egypt.

During 2019, molecular screening for avian influenza viruses (AIVs) was conducted in migratory birds in Egypt. During this screening, a total of thirty-four cloacal swab samples were collected from apparently healthy migratory birds (Anas acuta, Anas crecca, Rallus aquaticus, and Bubulcus ibis) (Table 1) from four Egyptian Governorates (Giza, Menoufia, Gharbia, and Dakahlia). Capturing by nets and sampling from live wild birds were carried out in accordance with all relevant guidelines, regulations and animal ethics permits issued by Veterinary Serum and Vaccine Research Institute (VSVRI), Agriculture Research Centre (ARC), Egypt. No clinical signs for AIV infection were observed in birds during sampling. Virus isolation for two passages was performed by inoculation into the allantoic cavity of 10- 
day old specific pathogen free (SPF) embryonated chicken eggs (ECEs) according to the OIE diagnostic procedures [29]. The allantoic fluids from inoculated eggs were assessed for agglutination activity via hemagglutination assay (HA) using $0.5 \%$ chicken erythrocytes according to OIE recommendation [29]. The positive HA samples were subjected to viral RNA extraction using a QIAamp viral RNA mini kit (Qiagen, Hilden, Germany) according to the manufacturer's protocol, then typed by $\mathrm{M}$ gene using RT-PCR [30]. The M gene positive samples were further subtyped for HA and NA genes as previously described [31, 32]. Meanwhile, all collected samples were screened for avian avulavirus and gammacoronavirus and were found negative.

The cDNA was synthesised by superscript III reverse transcriptase as per manufacturer protocol using the Uni-12 primer (5'-AGCRAAAGCAGG-3'). Genome was amplified as previously described [33] using the Q $5{ }^{\circledR}$ high-fidelity DNA polymerase package (NEB, UK).

\subsection{Sequencing and Sequence Analysis}

The PCR products were size separated by agarose gel electrophoresis, excised and purified from gels using the QIAquick Gel Extraction Kit (Qiagen, Hilden, Germany). The purified PCR products were used directly for sequencing using BigDye Terminator v3.1 Cycle Sequencing Kit (Applied Biosystems, California, USA) and ABI 3500 Genetic Analyzer (Life Technologies, California, USA) by Source Biosciences Sequencing Facility (Source Bioscience, UK).

Sequences generated in this study were submitted to the GenBank under accession numbers MT256069 and MT256070. The obtained sequences were subjected to NCBI BLASTN analysis (https://blast.ncbi.nlm.nih.gov/Blast.cgi). BioEdit 7.0 software was used for multiple sequences and genomic signature analysis [34]. The MEGA 7.0 was used for phylogenetic tree construction using the maximum likelihood method based on the General Time Reversible model, and Gamma distributed with invariable sites $(\mathrm{G}+\mathrm{I})$. The codon positions 1st, 2nd, 3rd, and non-coding sequences were included and the positions containing gaps and missing data were eliminated [35]. Analysis for the presence of glycosylation sites $\mathrm{N}-\mathrm{XT} / \mathrm{S}$ motif (X can be any amino acid except proline) within the HA protein using NetNGlyc 1.0 server (http://www.cbs.dtu.dk/services/NetNGlyc/). 


\subsection{Comparative Infectivity and Horizontal Transmission}

In the animal experiment, pathobiological assessments of cattle egret H5N8 strain (Cattle egret/Egypt/VRLCU/2019) using mock-infected, inoculated, and contact birds were performed individually (Fig 1) to study the pathobiology and possibility of horizontal transmission of wild-bird-origin H5N8 AIV to SPF chickens compared to chicken origin H5N8 AIV (MT256068 A/chicken/Egypt/VRLCU/2019 (H5N8) that was isolated from commercial layer flock vaccinated with inactivated commercial $\mathrm{H} 5 \mathrm{~N} 1$ vaccine.

The animal experiments were conducted in BSL3-compatible isolators in accordance with all relevant guidelines and animal ethics permits issued by Veterinary Serum and Vaccine Research Institute, Abbassia, Egypt. Chickens were kept on deep litter and water was provided through nipple drinkers/drinking towers and appropriate food was provided ad libitum.

Fifty SPF chickens (kindly provided by the Egyptian SPF Production Farm, Egypt) were housed separately in two groups; inoculated $(n=20)$ and non-inoculated $(n=30)$. Chickens in the non-inoculated group were divided into three further subgroups; two contact groups $(n=20$, 10 serve as contact group for group inoculated with cattle egret isolate while the other group serve as contact group for inoculated group with chicken isolate) and mock infected as negative control group ( $\mathrm{n}=10)$ (Fig 1). Chickens in the inoculated groups were divided into two groups; inoculated group with cattle egret H5N8 AIV and inoculated group with chicken origin H5N8 AIV. Inoculated chickens were inoculated with a dose of $100 \mu 1$ of $10^{6.5}$ EID $_{50} / \mathrm{ml}$ from both inoculated viruses through the intranasal route on day 21 old according to the standard procedures at VSVRI, Egypt. After $48 \mathrm{~h}$ post-challenge, the contact birds were introduced to both inoculated birds to assess for comparative infectivity and horizontal transmission of both viruses. The mock-infected group served as a negative control and was inoculated with $100 \mu \mathrm{l}$ of sterile normal saline.

For the next 14 days, all chickens were kept and monitored twice daily for clinical signs, including depression, anorexia, sneezing/coughing, respiratory disorders, ocular/ nasal discharge, conjunctivitis, ruffled feathers, reluctance to move and dyspnea along with collection of oropharyngeal and cloacal swabs to monitor shedding of the challenge viruses.

\subsection{Virus Shedding and Histopathology}

Swabs (oropharyngeal and cloacal) were examined for virus shedding $0,3^{\text {rd }}, 5^{\text {th }}, 7^{\text {th }}, 10^{\text {th }}$, and $14^{\text {th }}$ days post-inoculation (dpi) by using real-time RT-PCR as previously described [38]. The cycle threshold $(\mathrm{Ct})$ values were converted to egg infective dose $50\left(\mathrm{EID}_{50}\right)$ based on the 
standard curve $\left(\mathrm{R}^{2}>0.98\right)$. Different internal organs were collected from the euthanized/dead chickens in both the control and inoculated groups for detailed necropsy and histopathological examination. Selected tissues, including trachea, lung, liver, spleen and intestine, were collected and fixed by immersion in 10\% neutral buffered formalin at room temperature for $48 \mathrm{~h}$, followed by processing and embedding in paraffin wax. Tissue sections of $5 \mu \mathrm{m}$ were stained with Haematoxylin and Eosin and examined for microscopic lesions under a light microscope.

\subsection{Statistical Analysis}

Results of comparisons of inoculated groups were analysed using one-way analysis of variance (ANOVA), and significant differences among means were tested using Student's ttest. Kaplan-Meier analysis was performed to calculate the survival rate. All statistical tests were conducted using GraphPad Prism 7 (GraphPad Software, La Jolla, CA, USA). For all assays and comparisons, differences were considered significant at a $p$-value of $<0.05 .3$.

\section{Results}

\subsection{Screening of Samples and Virus Detection}

In this study, two out of thirty-four (2/34) cloacal swabs were positive for H5N8 subtype which were collected from four Egyptian governorates (Giza, Menoufia, Gharbia, and Dakahlia) during 2019. These two samples were negative for both H5N1 and H9N2 subtypes that are currently co-circulating in Egypt.

\subsection{Deduced Amino acids mutations analysis and Phylogeny}

The HA protein receptor-binding pocket of both wild bird origin H5N8 AIVs have characteristic features of avian receptor-specific binding affinity Q222 and G224. The multibasic cleavage site motif (PLREKRRKR\#GLF) in the HA proteins, characteristic for highly pathogenic AIVs, were identified in the H5N8 viruses. Analysis of glycosylation sites N-XT/S motif within the HA protein revealed that the two H5N8 isolates in this study carried six potential glycosylation sites at positions $10,23,165,286,483$ and 542 (H5 numbering after removal of the sequence encoding the signal peptide) similar to recently detected H5N8 viruses in poultry [16]. These N-linked glycosylation sites play an important role in the receptor- 
binding specificity which can affect or alter the antigenic sites on the HA protein leading to evolution of escape mutant strains.

Phylogenetic analysis of HA gene sequences revealed that Cattle egret/Egypt/VRLCU/2019 and Teal/Egypt/VRLCU/2019 viruses are clustered within the same genotype (Gt-5) of clade 2.3.4.4b, along with the Egyptian viruses recently detected from commercial chicken (Fig 2). Hassan et al. [20] have reported that Gt-5 have emerged since 2017 and circulated only in poultry while our results confirmed the circulation of this genotype within the wild birds.

\subsection{Comparative Pathogenicity between Wild bird and Chicken origin H5N8 strains}

In order to assess the potential impact of spillover of wild bird H5N8 viruses to poultry, it is imperative to investigate the infectious nature of isolates that were originated from nonchicken hosts and clustered within the H5N8 isolates from commercial chickens. For the inoculation experiment, mortality rate of the inoculated group with chicken H5N8 isolate was $100 \%$ (10/10) by day 7 dpi while its contact group was $100 \%(10 / 10)$ by day 14 dpi (Figure 3 ) which is statistically significant. However, the mortality rate of the inoculated group with cattle egret H5N8 isolate was $100 \%(10 / 10)$ by day 10 dpi while its contact group was $90 \%(9 / 10)$ by day 14 dpi (Fig 3); statistically significant. Clinical signs of H5N8 virus started to appear in both inoculated groups on the $3^{\text {rd }}$ day after virus inoculation with chicken origin H5N8 and on day 5 post inoculation with cattle egret-origin $\mathrm{H} 5 \mathrm{~N} 8$ isolate suggesting that the wild bird origin virus able to infect the chickens successfully. These clinical sings included depression, anorexia, respiratory disorders, oculonasal discharge, swelling of combs and wattles. Although severe clinical signs were more often observed in chickens inoculated with the chicken origin H5N8 isolate, the disease outcome was observed on day 3 dpi in both inoculated groups and was associated with the post-mortem lesions as congestion and inflammation of the trachea and lungs.

Our results revealed the direct correlation between high degree of virus shedding and level of transmissibility among chickens, which were $100 \%$ for chicken isolate and $90 \%$ for cattle egret isolate. Various tissues were collected from dead contact chickens that contained typical necropsy lesions that were absent in the mock-infected negative control group. Our results indicated that the H5N8 viruses are highly virulent, chicken-to-chicken transmissibility is high in case of spillover. 


\subsection{Comparative Virus shedding and Histopathological investigations}

Cloacal and oropharyngeal swabs were collected on alternate days until the end of the experiment. Viral shedding in cloacal and oropharyngeal swabs was observed for both inoculated groups on day 3 dpi, however, slight (non-significant) increase in virus shedding was noticed in the inoculated group with chicken $\mathrm{H} 5 \mathrm{~N} 8$ compared to cattle egret $\mathrm{H} 5 \mathrm{~N} 8$ isolate (Figure 4A and 4B). Meanwhile, virus shedding was detected in contact chickens of both inoculated groups and there was a slight increase in virus shedding in the contact group for chicken H5N8 compared to contact group for cattle egret H5N8 which is non-statistically significant and reached approximately the same titers on day 14 dpi (Fig 4A and 4B).

Severe histopathological alterations were observed in all internal organs in contact group of chicken $\mathrm{H} 5 \mathrm{~N} 8$ isolate in compare to wild bird origin $\mathrm{H} 5 \mathrm{~N} 8$ that might be the wild bird origin virus need additional time or further passage for adaptation to the poultry (Table 2). Trachea revealed focal necrosis of lamina epithelialis, edema in the lamina propria/ submucoas layer while lungs showed congestion of blood vessels, focal pulmonary hemorrhage, pneumonia characterized by infiltration of the air capillaries with inflammatory exudate (mainly mononuclear inflammatory cells) as well as focal pulmonary emphysema. Likewise, marked fibrinoid necrosis and lymphocytic necrosis and depletion were observed in the spleen. Caecal tonsils exhibited massive infiltration of the lamina propria with heterophils, with lymphocytic necrosis and depletion. In the liver, we observed Kupfer cell activation, dissociation of hepatic plates, cytoplasmic vacuolization of hepatocytes, and focal hepatic necrosis and apoptosis with infiltration by inflammatory cells, hyperplasia of the biliary epithelium, and fibroplasia in the portal triad (Fig 5).

\section{Discussion}

Wild birds are considered the natural reservoirs and the mixing vessel for avian influenza viruses, which play an important role in the viral diversity through generating novel reassortants [36- 38]. Previous studies have reported that circulating H5 and H7 LPAI viruses in gallinaceous poultry (chickens, turkeys, quail, etc.) could be able to mutate to produce highly pathogenic avian influenza viruses that can cause severe systemic disease and high mortality in gallinaceous poultry and are typically easily transmissible among [39].

Due to the ability of clade 2.3.4.4 viruses to reassort, a variety of different genotypes and subtypes has been emerged during their spread from Asia to Europe and Africa [6, 7]. The key mechanisms for the continuous evolution of new subtypes and genotypes of avian influenza 
viruses are underlined by the aggregation of random mutations and genes reassortment [28]. Recently, HPAI H5N8 viruses have been involved in multiple independent reassortment events with other AIV subtypes, with variable genetic diversity at the subclade level; have been reported from several Middle East, European and Asian countries [6, 7, 9, 11, 12]. Therefore, tracking and characterization of emerging influenza viruses in wild birds and commercial poultry is a plausible approach to track their zoonotic potential. The first HPAI H5N8 outbreak in Egypt was detected in wild birds, which had a closer genetic relatedness to European viruses circulating at that time [11]. Therefore, the influenza epidemiological situation in Egypt became complicated especially with the co-circulation of both HPAIV H5N1 clade 2.2.1.2, clade 2.2.1.1 and LPAIV H9N2 among different poultry species [40, 41].

Previous studies reported that Egyptian H5N8 AIVs of clade 2.3.4.4b can be diversified into at least 5 genotypes (Gt1- Gt5) [20]. Interestingly, Gt-3 and -4 bear gene fragments of their genome that were closely similar to those in LPAIV H7N9 and H7N3 and H3N6 viruses or concurrently in Egyptian wild birds [12, 16, 19, 20]. Moreover, it was thought that reassortant avian influenza viruses have been developed, adapted, and gained dominance over the previously circulating genotypes in the Egyptian poultry flocks as a result of unknown selective gain [21]. The role of the genetic makeup of wild bird origin influenza viruses in pathobiology remains unclear. Here, to assess the potential role of wild birds in disseminating HPAI viruses compare to poultry H5N8 isolate, we compared the pathogenicity, viral shedding patterns, and transmissibility of wild bird-origin H5N8 HPAI virus of clade 2.3.4.4 in chickens. Although data are limited, some H5/H7 HPAI viruses can adapt to Gallinaceous species and less likely cause disease in wild birds $[42,43]$. Analysis of the deduced residues at the cleavage site of characterized isolates in the current study and previously reported isolates from Egypt [12- 21].

Our previous studies demonstrated the importance of wild birds not only for introducing the influenza viruses but also other avian respiratory viruses [44]. Furthermore, comparative analysis of functional domains of HA proteins of studied isolates highlight evolutionary constrains on the HA protein especially as there are no vaccination programs against $\mathrm{H} 5 \mathrm{~N} 8$ in the Egyptian poultry industry until now. Only vaccination against H5N1 and H9N2 AIVs are being practiced in the country. Previous findings showed that most of the available commercial poultry H5 vaccines used among the Egyptian poultry industry were ineffective against challenge with $\mathrm{H} 5 \mathrm{~N} 8$ viruses because of the genetic difference between the seed viruses in those vaccines and the H5N8 viruses currently circulating in Egypt [45]. Likewise, the genetic diversity and low reactions between Egypt's H5 commercial vaccines and current $\mathrm{H} 5 \mathrm{~N} 8$ viruses 
show that the vaccines might not be successful in the field, or only implement partial safety, and thus may lead to escape mutant strains induced by vaccines.

Taken together, nucleotide and subsequent amino acid substitutions explained the evolving nature of RNA viruses [46], especially influenza viruses that warrant future investigation to delineate the importance of these mutations in the pathobiology of these viruses especially in the wild birds-driven potential spillover events. Phylogenetic analysis of the full-length HA gene of two wild bird-originated H5N8 AIVs (A/Cattle egret/Egypt/VRLCU/2019 and $\mathrm{A} /$ Teal/Egypt/VRLCU/2019) revealed close relationships to Gt-5 of clade 2.3.4.4b, which is predominantly circulating among the Egyptian poultry sectors since 2017 [20] and were found to be closely related to isolates recently reported from Egyptian commercial chickens. These results highlight the risk of $\mathrm{H} 5 \mathrm{~N} 8 \mathrm{AIVs}$ transmission among different avian hosts especially with the high reporting cases of H5N8 compare to H5N1 and H9N2 AIVs. Meanwhile, these genetic and phylogenetic features of the HA genes propose the intercontinental dissemination of HPAIV (H5N8) through wild birds and its potential introduction in the Egypt [12]. Cattle egret is a native feral bird commonly seen in Egypt; therefore, we propose two possibilities for infection of cattle egret with H5N8. Firstly, due to direct and/or indirect contact with other infected wild bird with H5N8. Secondly, as a result with indirect contact with manure of infected chickens.

The current study aims to assess the clinicopathological impact of two selected H5N8 isolates (chicken-origin and wild bird-origin) to properly evaluate the infectivity and transmissibility of both isolates, including onset of clinical signs, mortality, virus shedding and transmission to healthy chickens. The oculonasal route was used to induce infection within the inoculated groups as a natural pathway of infection under field conditions [32]. We observed sudden deaths in inoculated chickens, which was expected, with severe respiratory clinical signs and sudden death which is characteristic for highly pathogenic avian influenza viruses infection. Similar observations have been reported [47, 48] in immunologically naive birds inoculated with H5N8 AIVs. Meanwhile, severe form of infection was observed in chickens upon infection with chicken origin H5N8 compared to cattle egret origin H5N8. Although severity of observed respiratory clinical signs was relatively less severe for Cattle egret/Egypt/VRLCU/2019 isolate than chicken/Egypt/VRLCU/2019 isolate, the morbidity, mortality rates and virus shedding were comparable between these groups. Mortality rate for the inoculated group with chicken H5N8 isolate was $100 \%$ by day 7 dpi while its contact group was $100 \%$ by day 14 dpi. However, complete death (100\%) for the inoculated group with cattle 
egret H5N8 isolate by day 10 dpi was noticed compared to $90 \%$ in the contact group by day 14 dpi.

Viral titers in oropharyngeal and cloacal swabs elucidated the aforementioned pathogenicity variation of the two isolates and coincided with their replication in the respiratory and intestinal tracts. Viral shedding was detected in both cloacal and oropharyngeal swabs from inoculated and contact groups of cattle egret H5N8 on day 3 post infection, which indicate the ability of wild bird-origin H5N8 virus to replicate efficiently in chickens.

\section{Conclusions}

The generated data in our study can be informative for AIV control strategies for the Egyptian administrative authorities. Our study demonstrates the genetic characterization of H5N8 viruses in cattle egret and teal birds with high similarity. Both isolate clustered with previously characterized H5N8 viruses from both Egyptian wild birds and commercial poultry. In addition, our findings confirmed the possibility for transmission of cattle egret origin H5N8 virus to chicken that might be helpful to improve the prevention strategies. Continuous disease monitoring, surveillance and subsequent genome-based characterization are essential to assess the spillover events. While vaccines are key elements for influenza control, these require frequent evaluation and upgradation. Therefore, an assessment of coverage and efficacy of different vaccines and or vaccination policy in the field conditions should be reconsidered along with strict biosecurity measures.

To recapitulate, existing disease surveillance and control strategies could be revised in disease-endemic settings. It is also government and public duty to disseminate information and legislation regarding the safe care of both domestic and natural animals in order to minimize bird-to-human transmission. It is advisable to embrace scenario in case of spillover over of H5N8 AIVs from wild birds to commercial poultry and reverse spillover possibility from commercial poultry to wild birds, which will help in preparing a comprehensive plan focusing on increased surveillance and the actions to be taken to prevent its spread.

\section{Declaration of Competing Interest}

The authors declare no conflict of interest.

\section{Acknowledgements}

This work was financed by International Foundation for Science (IFS) (project No. I3-B-6270-1), The Organisation of Islamic Cooperation's Standing Committee on Scientific and 
Technological Cooperation (COMSTECH). The funding sources had no role in the study design, collection, or analysis of the data, writing of the manuscript, or in the decision to submit the manuscript for publication.

\section{References}

[1] J.D. Alexander, Brown, I.H. History of highly pathogenic avian influenza. Rev. Sci. Tech. 28 (2009), 19-38. https://doi.org/10.20506/rst.28.1.1856

[2] M. Gu, W. Liu, Y. Cao, D. Peng, X. Wang, H. Wan, G. Zhao, Q. Xu, W. Zhang, Q. Song, Y. Li, X. Liu. Novel reassortant highly pathogenic avian influenza (H5N5) viruses in domestic ducks, China. Emerg. Infect. Dis. 17(2011), 1060-1063. https://doi.org/10.3201/eid/1706.101406

[3] K. Zhao, M. Gu, L. Zhong, Z. Duan, Y. Zhang, Y. Zhu, G. Zhao, M. Zhao, Z. Chen, S. Hu, W. Liu, X. Liu, D. Peng, X. Liu. Characterization of three H5N5 and one H5N8 highly pathogenic avian influenza viruses in China. Vet Microbiol. 163 (2013):351-7. https://doi.org/10.1016/j.vetmic.2012.12.025

[4] H. Wu, X. Peng, L. Xu, C. Jin, L. Cheng, X. Lu, T. Xie, H. Yao, N. Wu. Novel reassortant influenza $\mathrm{A}(\mathrm{H} 5 \mathrm{~N} 8)$ viruses in domestic ducks, eastern China. Emerg. Infect. Dis. 20( 2014):1315-8. https://doi.org/10.3201/eid2008.140339

[5] C. Abolnik. Outbreaks of Clade 2.3.4.4 H5N8 highly pathogenic avian influenza in 2018 in the northern regions of South Africa were unrelated to those of 2017. Transbound. Emerg. Dis. 67(2020) 1371-1381. https://doi.org/10.1111/tbed.13448

[6] EFSA Panel on Animal Health and Welfare (AHAW), S. More, D. Bicout, A. Bøtner, A. Butterworth, P. Calistri, K. Depner, S. Edwards, B. Garin-Bastuji, M. Good, C. Gortázar Schmidt, V. Michel, M.A. Miranda, S.S. Nielsen, M. Raj, L. Sihvonen, H. Spoolder, H.H. Thulke, A. Velarde, P. Willeberg, C. Winckler, A. Breed, A. Brouwer, M. Guillemain, T. Harder, I. Monne, H. Roberts, F. Baldinelli, F. Barrucci, C. Fabris, L. Martino, O. MosbachSchulz, F. Verdonck, J. Morgado, J.A. Stegeman. Avian influenza. EFSA J. 15 (2017):e04991. https://doi.org/10.2903/j.efsa.2017.4991

[7] A. Pohlmann, E. Starick, T. Harder, C. Grund, D. Höper, A. Globig, C. Staubach, K. Dietze, G. Strebelow, R.G. Ulrich, J. Schinköthe, J.P. Teifke, F.J. Conraths, T.C. Mettenleiter, M. Beer. Outbreaks among wild birds and domestic poultry caused by reassorted influenza A (H5N8) 
404

405

406

407

408

409

410

411

412

413

414

415

416

417

418

419

420

421

422

423

424

425

426

427

428

429

430

431

432

433

Clade 2.3.4.4 Viruses, Germany, 2016. Emerg. Infect. Dis. 23 (2017):633-636. https://doi.org/10.3201/eid2304.161949

[8] L. Dong-Hun, S. Kirill, E.S. David, K. Olga, S. Ivan, M. Kabilov, A. Alekseev, V. Irza, A. Shestopalov. Novel Reassortant Clade 2.3.4.4 Avian Influenza A(H5N8) Virus in Wild Aquatic Birds, Russia, 2016. Emerg. Infect. Dis. 23 (2017) 359-360. https://doi.org/10.3201/eid2302.161252.

[9] T. Harder, S. Maurer-Stroh, A. Pohlmann, E. Starick, D. Höreth-Böntgen, K. Albrecht, G. Pannwitz, J. Teifke, V. Gunalan, R.T. Lee, C. Sauter-Louis, T. Homeier, C. Staubach, C. Wolf, G. Strebelow, D. Höper, C. Grund, F.J. Conraths, T.C. Mettenleiter, M. Beer. Influenza A(H5N8) Virus Similar to Strain in Korea Causing Highly Pathogenic Avian Influenza in Germany. Emerg Infect Dis. 2015 May;21(5):860-3. https://doi.org/10.3201/eid2105.141897.

[10] S. Nagarajan, M. Kumar, H.V. Murugkar, S. Tripathi, S. Shukla, S. Agarwal, G. Dubey, R.S. Nagi, V.P. Singh, C. Tosh. Novel Reassortant Highly Pathogenic Avian Influenza (H5N8) Virus in Zoos, India. Emerg. Infect. Dis. 23 (2017) 717-719. https://doi.org/10.3201/eid2304.161886

[11] A. Fusaro, I. Monne, P. Mulatti, B. Zecchin, L. Bonfanti, S. Ormelli, A. Milani, K. Cecchettin, P. Lemey, A. Moreno, P. Massi, T. Dorotea, S. Marangon, C. Terregino. Genetic Diversity of Highly Pathogenic Avian Influenza A(H5N8/H5N5) Viruses in Italy, 2016-17. Emerg. Infect. Dis. 23 (2017):1543-1547. https://doi.org/10.3201/eid2309.170539

[12] N. Yehia, M.M. Naguib, R. Li, N. Hagag, M. El-Husseiny, Z. Mosaad, A. Nour, N. Rabea, W. Hasan, M. Hassan, T. Harder, A. Arafa. Multiple introductions of reassorted highly pathogenic avian influenza viruses (H5N8) clade 2.3.4.4b causing outbreaks in wild birds and poultry in Egypt. Infect. Genet. Evol. $58 \quad$ (2018) 56-65. https://doi.org/10.1016/j.meegid.2017.12.011

[13] A. Kandeil, A. Kayed, Y. Moatasim, R.J. Webby, P.P. McKenzie, G. Kayali, M.A. Ali. Genetic characterization of highly pathogenic avian influenza A H5N8 viruses isolated from wild birds in Egypt. J. Gen. Virol. 98 (2017) 1573-1586. https://doi.org/10.1099/jgv.0.000847 [14] A.A. Selim, A.M. Erfan, N. Hagag, A. Zanaty, A.H. Samir, M. Samy, A. Abdelhalim, A.A. Arafa, M.A. Soliman, M. Shaheen, E.M. Ibraheem, I. Mahrous, M.K. Hassan, M.M. Naguib. Highly pathogenic avian influenza virus (H5N8) clade 2.3.4.4 infection in migratory 
434 birds, Egypt. Emerg. Infect. Dis. $23 \quad$ (2017) 1048-1051. 435 https://doi.org/10.3201/eid2306.162056

436 [15] A.H. Salaheldin, H.S. El-Hamid, A.R. Elbestawy, J. Veits, H.M. Hafez, T.C. Mettenleiter, 437 E.M. Abdelwhab. Multiple Introductions of Influenza A (H5N8) Virus into Poultry, Egypt, 438 2017. Emerg. Infect. Dis. 24 (2018) 943-6. https://doi.org/10.10.3201/eid2405.171935

439 [16] K.E. Hassan, M.F. El-Kady, A.A.A. El-Sawah, C. Luttermann, R. Parvin, S. Shany, M. 440 Beer, T Harder. Respiratory disease due to mixed viral infections in poultry flocks in Egypt 441 between 2017 and 2018: Upsurge of highly pathogenic avian influenza virus subtype H5N8 442 since 2018. Transbound. Emerg. Dis. 2019; https://doi.org/doi.org/10.1111/tbed.13281

443 [17] A. Kandeil, J.T. Hicks, S.G. Young, A.N. El Taweel, A.S. Kayed, Y. Moatasim, O. Kutkat, 444 O. Bagato, P.P. McKenzie, Z. Cai, R. Badra, M. Kutkat, J. Bahl, R.J. Webby, G. Kayali, M.A. 445 Ali. Active surveillance and genetic evolution of avian influenza viruses in Egypt, 2016-2018. 446 Emerg. Microbes Infect. 8 (2019) 1370-1382. https://doi.org/10.1080/22221751.2019.1663712

447 [18] M.M. Naguib, J.H. Verhagen, A. Samy, P. Eriksson, M. Fife, A. Lundkvist, P. Ellström, 448 J.D. Järhult. Avian influenza viruses at the wild-domestic bird interface in Egypt. Infect. Ecol. Epidemiol. 9 (2019) 1575687. https://doi.org/10.1080/20008686.2019.1575687

[19] A.A. Shehata, M.E. Sedeik, A.R. Elbestawy, M.A. Zain El-Abideen, H.H. Ibrahim, W.H. Kilany, A. Ali. Co-infections, genetic, and antigenic relatedness of avian influenza H5N8 and H5N1 viruses in domestic and wild birds in Egypt. Poult. Sci. 98 (2019) 2371-2379. https://doi.org/10.10.3382/ps/pez011.

[20] K.E. Hassan, N. Saad, H.H. Abozeid, S. Shany, M.F. El-Kady, A. Arafa, A.A.A. ElSawah, F. Pfaff, H.M. Hafez, M. Beer. Genotyping and reassortment analysis of highly 456 pathogenic avian influenza viruses H5N8 and H5N2 from Egypt reveals successive annual replacement of genotypes. Infect. Genet. Evol. 84 (2020), 104375. https://doi.org/10.1016/j.meegid.2020.104375.

[21] N.M. Hagag, A.M. Erfan, M. El-Husseiny, A.G. Shalaby, M.A. Saif, M.M. Tawakol, A.A. Nour, A.A. Selim, A.S. Arafa, M.K. Hassan, W.M.M. Hassan, H.A. Fahmy, E. Ibraheem, M. Attia, A.M.M. Abdelhakim, M.A. Shahein, M.M. Naguib. Isolation of a Novel Reassortant Highly Pathogenic Avian Influenza (H5N2) Virus in Egypt. Viruses. 11 (2019):565. https://doi.org/10.3390/v11060565. 
[22] D.H. Lee, M.K. Torchetti, J. Hicks, M.L. Killian, J. Bahl, M. Pantin-Jackwood, D.E.

Swayne. Transmission Dynamics of Highly Pathogenic Avian Influenza Virus A(H5Nx) Clade

2.3.4.4, North America, 2014-2015. Emerging. infectious diseases. 24 (2018), 1840-1848.

[23] T. Saito, T. Tanikawa, Y. Uchida, N. Takemae, K. Kanehira, R. Tsunekuni. Intracontinental and intercontinental dissemination of Asian H5 highly pathogenic avian influenza virus (clade 2.3.4.4) in the winter of 2014-2015. Rev Med Virol. 25 (2015) 388-405. https://doi.org/10.1002/rmv.1857

472 [24] N.J. Hill, I.T. Hussein, K.R. Davis, E.J. Ma, T.J. Spivey, A.M. Ramey, W.B. Puryear, 473 S.R. Das, R.A. Halpin, X. Lin, N.B. Fedorova, D.L. Suarez, W.M. Boyce, J.A. Runstadler. 474 Reassortment of Influenza A Viruses in Wild Birds in Alaska before H5 Clade 2.3.4.4 Outbreaks. Emerg. Infect. Dis. 23 (2017):654-657. https://doi.org/10.3201/eid2304.161668 [25] M.J. Poen, J.H. Verhagen, R.J. Manvell, I. Brown, T.M. Bestebroer, S. van der Vliet, O. Vuong, R.D. Scheuer, H.P. van der Jeugd, B.A. Nolet, E. Kleyheeg, G.J. Müskens, F.A. Majoor, C. Grund, R.A. Fouchier. Lack of virological and serological evidence for continued circulation of highly pathogenic avian influenza H5N8 virus in wild birds in the Netherlands, 14 November 2014 to 31 January 2016. Euro Surveill. 2016, 21(38):30349. https://doi.org/10.2807/1560-7917.ES.2016.21.38.30349.

[26] E.M. Abdelwhab, H.M Hafez. An overview of the epidemic of highly pathogenic H5N1 avian influenza virus in Egypt: epidemiology and control challenges. Epidemiol Infect. 139 (2011):647-657. https://doi.org/10.1017/S0950268810003122

[27] P. Denny. Africa. In: Finlayson M, Moser M, eds. Wetlands. London, UK: International Waterfowl and Wetlands Research Bureau; 1991:115-148

487 [28] M.M. Naguib, A.S. Arafa, M.F. El-Kady, A.A. Selim, V. Gunalan, S. Maurer-Stroh, K.V. 488 Goller, M.K. Hassan, M. Beer, E.M. Abdelwhab, T.C. Harder. Evolutionary trajectories and 489 diagnostic challenges of potentially zoonotic avian influenza viruses H5N1 and H9N2 cocirculating in Egypt. Infect. Genet. Evol. 34 (2015) 278-91. https://doi.org/10.1016/j.meegid.2015.06.004. 
492 [29] OIE: Chapter 2.3.4. Avian influenza. Available online at: 493 http://www.oie.int/fileadmin/Home/eng/Health_standards/tahm/2.03.04_AI.pdf (Accessed 22 494 Nov. 2014).

495 [30] Naguib MM, Graaf A, Fortin A, Luttermann C, Wernery U, Amarin N, Hussein HA, H. 496 Sultan, B. Adhadh, M.K. Hassan, M. Beer, I. Monne, T.C. Harder. Novel real-time PCR-based patho- and phylotyping of potentially zoonotic avian influenza A subtype H5 viruses at risk of incursion into Europe in 2017. Euro Surveill. 22 (2017). pii: 30435. https://doi.org/10.2807/1560-7917.ES.2017.22.1.30435.

500 [31] I. Monne, S. Ormelli, A. Salviato, C. De Battisti, F. Bettini, A. Salomoni, A. Drago, B. 501 Zecchin, I. Capua, G. Cattoli. Development and validation of a one-step real-time PCR assay for simultaneous detection of subtype H5, H7, and H9 avian influenza viruses. J. Clin. Microbiol. 46 (2008) 1769- 1773. https://doi.org/10.1128/JCM.02204-07.

[32] E. Spackman, D.L. Suarez. Type A influenza virus detection and quantitation by real-time 505 RT-PCR. Methods Mol. Biol. 436 (2008) 19-26. https://doi.org/10.1007/978-159745-279-3 4

[33] E. Hoffmann, J. Stech, Y. Guan, R.G. Webster, D.R. Perez. Universal primer set for the 507 full-length amplification of all influenza A viruses. Arch. Virol. 146 (2001) 2275-2289. https://doi.org/10.1007/s007050170002.

[34] T.A. Hall. BioEdit: a user-friendly biological sequence alignment editor and analysis for Windows 95/98/NT. Nucleic Acids Symp. 41 (1999) 95-98.

[35] S. Kumar, G. Stecher, K. Tamura. MEGA7: molecular evolutionary genetics analysis version 7.0 for bigger datasets. Mol. Biol. Evol. 33 (2016) 1870-1874. https://doi.org/10.1093/molbev/msw054

514 [36] N.J. Hill, I.T. Hussein, K.R. Davis, E.J. Ma, T.J. Spivey, A.M. Ramey, W.B. Puryear, S.R. 515 Das, R.A. Halpin, X. Lin, N.B. Fedorova, D.L. Suarez, W.M. Boyce, J.A. Runstadler. 516 Reassortment of Influenza A Viruses in Wild Birds in Alaska before H5 Clade 2.3.4.4 517 Outbreaks. Emerg. Infect. Dis. 23 (2017) 654-657. https://doi.org/10.3201/eid2304.161668.

518 [37] N.S. Lewis, J.H. Verhagen, Z. Javakhishvili, C.A. Russell, P. Lexmond, K.B. Westgeest, 519 T.M. Bestebroer, R.A. Halpin, X. Lin, A. Ransier, N.B. Fedorova, T.B. Stockwell, N. Latorre520 Margalef, B. Olsen, G. Smith, J. Bahl, D.E. Wentworth, J. Waldenström, R.A.M. Fouchier, M. 521 de Graaf. Influenza A virus evolution and spatio-temporal dynamics in Eurasian wild birds: a 
522

523

524

525

526

527

528

529

530

531

532

533

534

535

536

537

538

539

540

541

542

543

544

545

546

547

548

549

550

phylogenetic and phylogeographical study of whole-genome sequence data. J. Gen. Virol. 96 (2015) 2050-2060. https://doi.org/10.1099/vir.0.000155.

[38] L. Lu, S.J. Lycett, A.J. Leigh Brown. Reassortment patterns of avian influenza virus internal segments among different subtypes. BMC Evol. Biol. 14 (2014) 16. https://doi.org/10.1186/1471-2148-14-16.

[39] D.E. Swayne, M.J. Pantin-Jackwood. Pathobiology of avian influenza virus infections in birds and mammals, p 87-122. In Swayne DE (ed), Avian influenza. Blackwell Publishing, Ames, IA; 2008.

[40] M.A. Rohaim, R.F. El-Naggar, M.M. Hamoud, S.A. Nasr, E. Ismael, S.E. Laban, A.A. Ahmed, M. Munir. Re-Emergence of a Novel H5N1 Avian Influenza Virus Variant Subclade 2.2.1.1 in Egypt During 2014. Transbound. Emerg. Dis. 64 (2017) 1306-1312. https://doi.org/10.1111/tbed.12472

[41] G. Kayali, A. Kandeil, R. El-Shesheny, A.S. Kayed, A.M. Maatouq, Z. Cai, P.P. McKenzie, R.J. Webby, S. El Refaey, A. Kandeel, M.A. Ali. Avian Influenza A(H5N1) Virus in Egypt. Emerg. Infect. Dis. 22 (2016) 379-388. https://doi.org/10.3201/eid2203.150593.

[42] G.W. Wood, G. Parsons, D.J. Alexander. Replication of influenza A viruses of high and low pathogenicity for chickens at different sites in chickens and ducks following intranasal inoculation. Avian Pathol. 24 (1995) 545-551. https://doi.org/10.1080/03079459508419093.

[43] D.E. Swayne. Epidemiology of avian influenza in agricultural and other man-made systems, p 59-85. In Swayne DE (ed), Avian influenza. Blackwell Publishing, Ames, IA; 2008. [44] M.A. Rohaim, R.F. El Naggar, A.M. Helal, M.M. Bayoumi, M.A. El-Saied, K.A. Ahmed, M.Z. Shabbir, M. Munir. Genetic Diversity and Phylodynamics of Avian Coronaviruses in Egyptian Wild Birds. Viruses 11 (2019) 57. https://doi.org/10.3390/v11010057

[45] A. Kandeil, J.S.M. Sabir, A. Abdelaal, E.H. Mattar, A.N. El-Taweel, M.J. Sabir, A.A. Khalil, R. Webby, G. Kayali, M.A. Ali. Efficacy of commercial vaccines against newly emerging avian influenza H5N8 virus in Egypt. Sci Rep. 2018 Jun 26;8(1):9697. https://doi.org/10.1038/s41598-018-28057-x.

[46] A.S. Lauring, R. Andino. Quasispecies Theory and the Behavior of RNA Viruses. PLoS Pathog. 6 (2010): e1001005. https://doi.org/10.1371/journal.ppat.1001005 
551 [47] D. Lee, J. Kwon, J. Noh, J. Park, S. Yuk, T. Erdene-Ochir, J. Lee, S. Park, I. Choi, S. Lee,

552 C. Song. Pathogenicity of the Korean H5N8 highly pathogenic avian influenza virus in 553 commercial domestic poultry species. Avian Pathol. 45 (2016) 208-211.

554 https://doi.org/10.1080/03079457.2016.1142502

555 [48] M.S. Imran, A. Aslam, M. Yasin, T. Yaqoob, B. Zahid. A Comparative Assessment of 556 Efficacy of Currently Applied Vaccines in Broiler Chicken against Individual and Co-Infection 557 with Field Prevailing Newcastle Disease and Infectious Bronchitis Viruses. Pakistan J. Zool., 558202052 (5) 1895-1901. https://dx.doi.org/10.17582/journal.pjz/20190424090449

559

560

561

562

563

564

565

566

567

568

569

570

571

572

573

574

575

576

577

578

579 


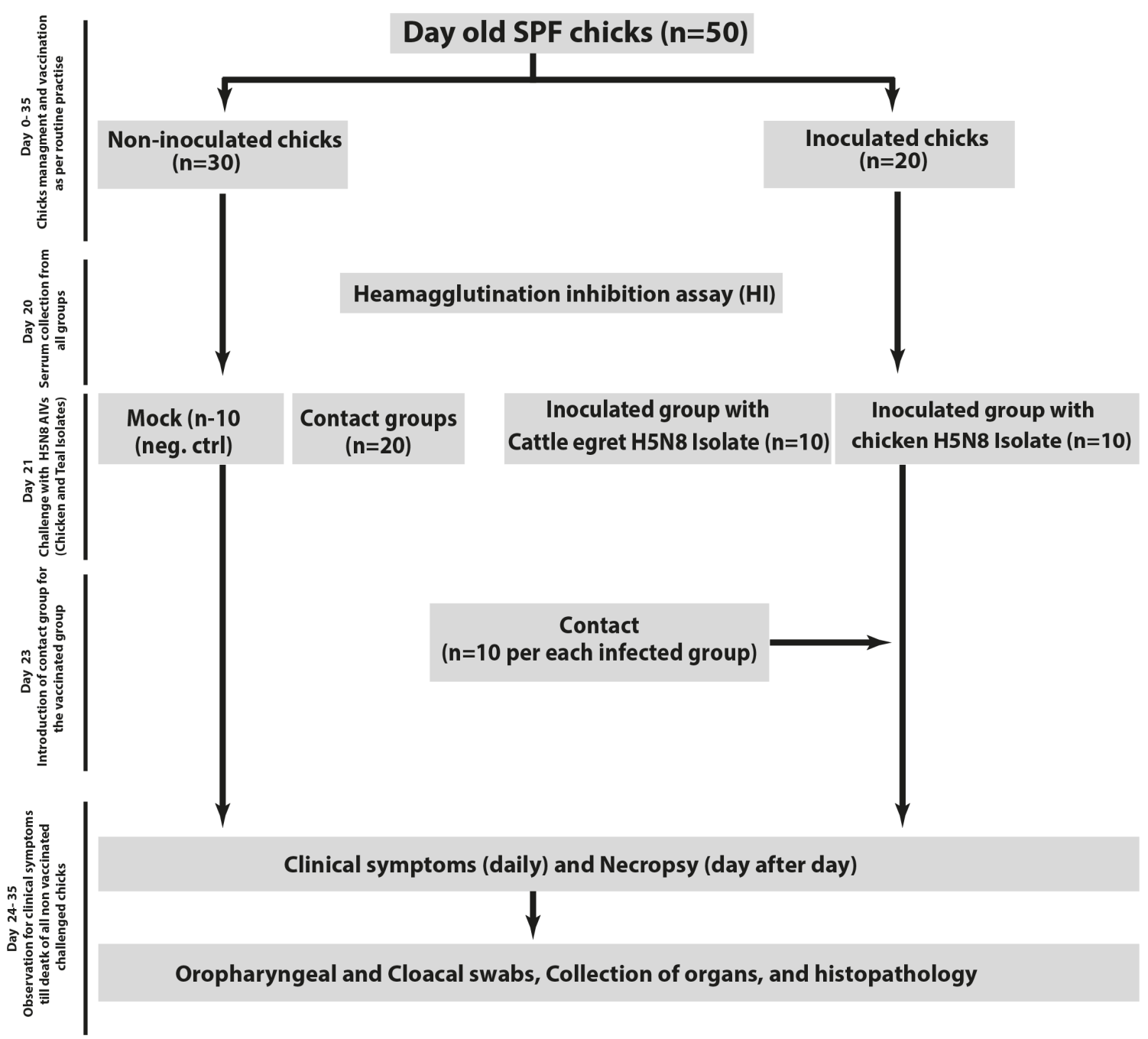

Fig 1. Experimental plan for the assessment of virus infectivity and transmission in chickens. 


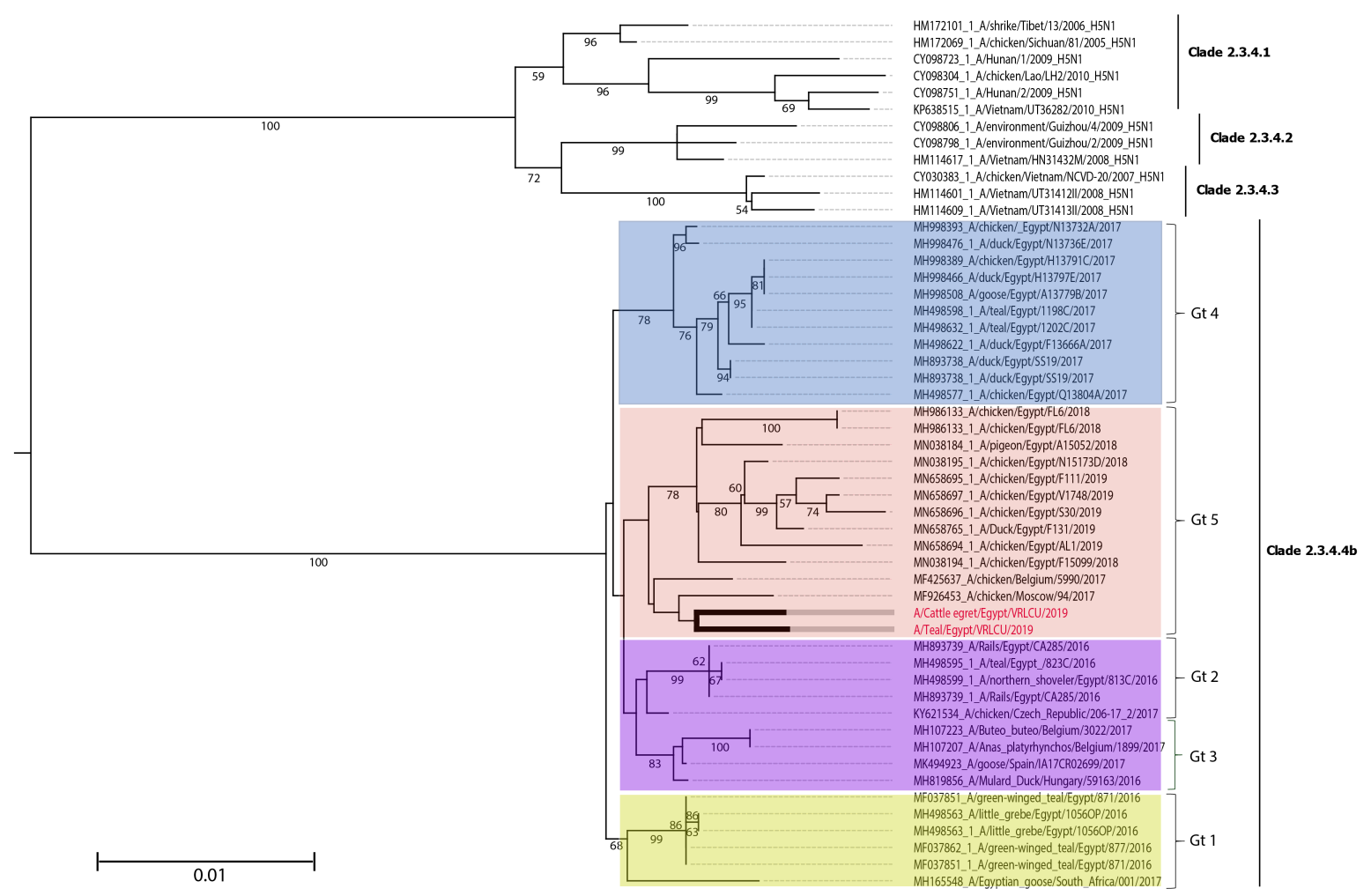

591

592 Fig 2. Phylogenetic analysis of studied isolates and their clustering patterns with representative

593 avian influenza clades. Full length HA gene based phylogenetic analysis of two wild bird origin

594 H5N8 isolates with representative strains of clade 2.3.4.4b currently circulating in Egypt. The 595 reported isolates clustered within Gt-5 of clade 2.3 .4 .4b with close relationship with the recent 596 characterized strains from Egyptian commercial poultry and wild birds.

597

598

599

600

601 


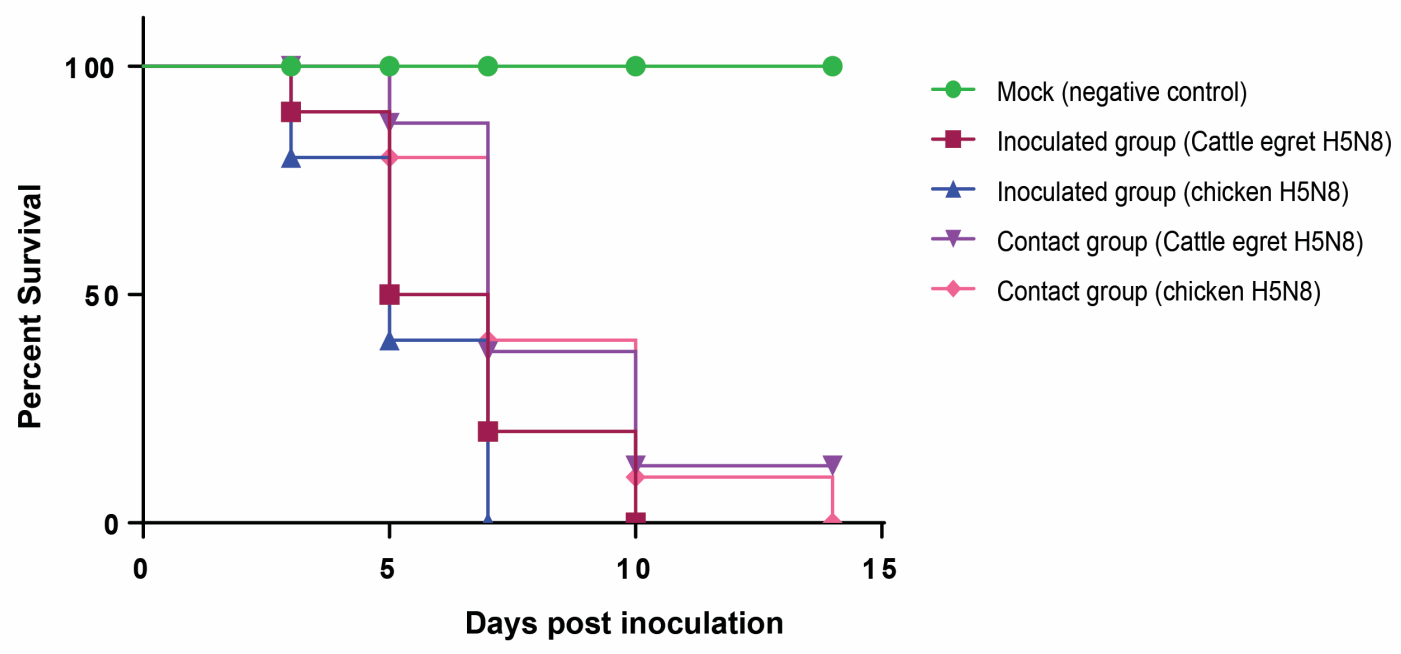

605

606 Fig 3. Percentage survival rates of negative control group, infected group with cattle egret 607 isolate and its contact group compared to infected group with chicken origin H5N8 and its 608 contact group.

609

(A)

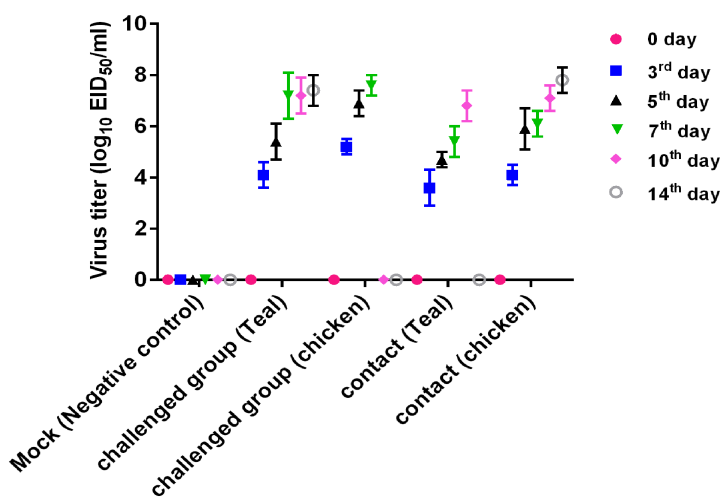

(B)

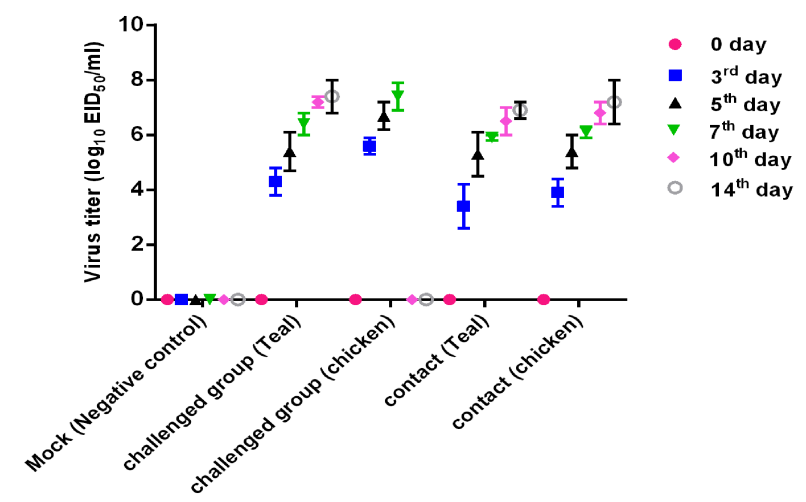

611 Fig 4. Infectivity, and Evaluation of viral shedding from A) oropharyngeal and B) cloacal 612 swabs for chickens inoculated with Cattle egret/Egypt/VRLCU/2019 and 613 chicken/Egypt/VRLCU/2019 H5N8 viruses. Each data point represents the virus titers 


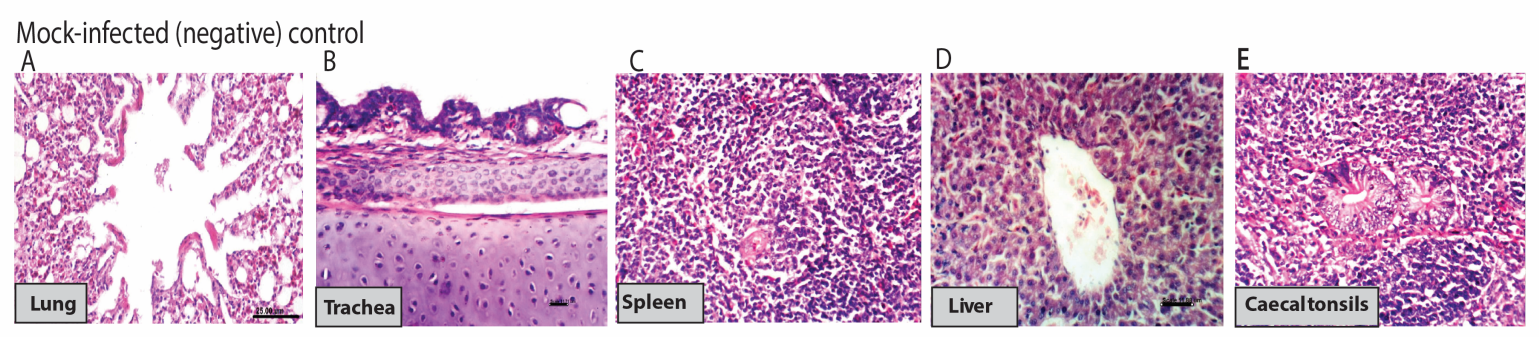

A/Cattle egret/Egypt/VRLCU/2019 (H5N8)
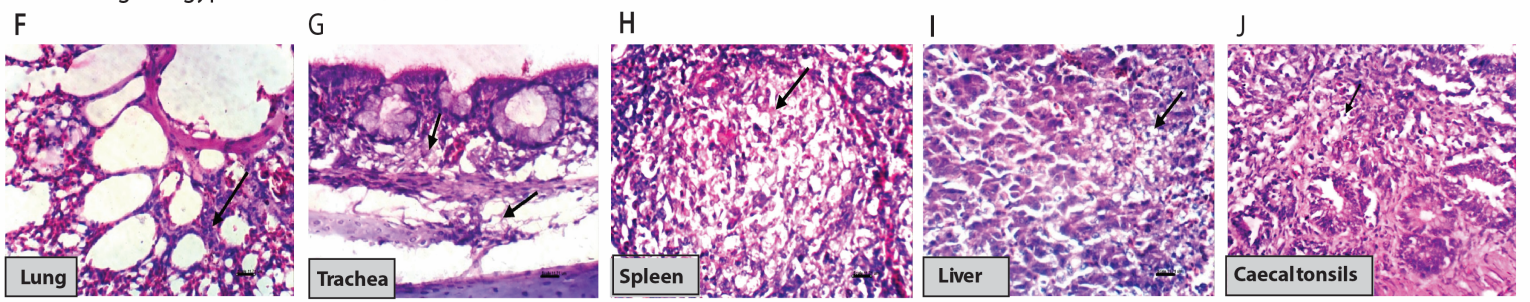

A/chicken/Egypt/VRLCU/2019 (H5N8)
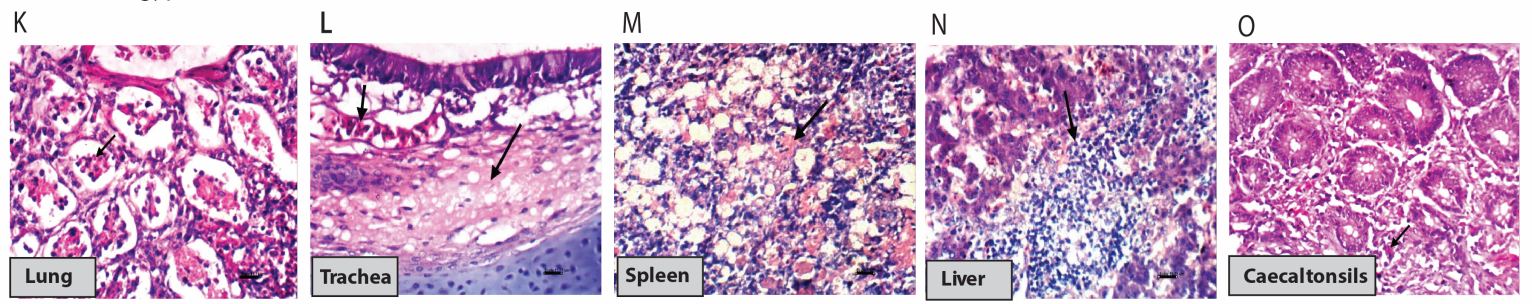

618

619 Fig 5. Microscopic examination of different tissues collected from chicken inoculated with

620 Cattle egret/Egypt/VRLCU/2019 and chicken/Egypt/VRLCU/2019 isolates. Arrows

621 indicate lesions in the affected tissues. (A,B,C,D,E and F) Histology of normal tissues:

622 lung, trachea, spleen, liver and caecal tonsil collected from the mock-infected group.

623 (F,G,H,I and J) Histopathological lesions in chicken tissues inoculated with cattle egret 624 isolate. (K,L,M,N and O) histopathological lesions in different tissues collected from 625 chickens which were inoculated with chicken isolate. 
630

631

632

633

634

635

636

637 